\title{
Sequence of post-moult exoskeleton hardening preserved in a trilobite mass moult assemblage from the Lower Ordovician Fezouata Konservat-Lagerstätte, Morocco
}

\author{
Harriet B. Drage, Thijs R.A. Vandenbroucke, Peter Van Roy, and Allison C. Daley \\ Acta Palaeontologica Polonica 64 (2), 2019: 261-273 doi:https://doi.org/10.4202/app.00582.2018
}

Euarthropods have a tough exoskeleton that provides crucial protection from predation and parasitism. However, this is restrictive to growth and must be periodically moulted. The moulting sequence is well-known from extant arthropods, consisting of: (i) the long inter-moult stage, in which no changes occur to the hardened exoskeleton; (ii) the pre-moult stage where the old exoskeleton is detached and the new one secreted; (iii) exuviation, when the old exoskeleton is moulted; and (iv) the post-moult stage during which the new exoskeleton starts as soft, thin, and partially compressed and gradually hardens to the robust exoskeleton of the inter-moult stage. Trilobite fossils typically consist of inter-moult carcasses or moulted exuviae, but specimens preserving the post-moult stage are rare. Here we describe nine specimens assigned to Symphysurus ebbestadi representing the first group of contemporaneous fossils collected that preserve all key stages of the moulting process in one taxon, including the post-moult stage. They were collected from a single lens in the Tremadocian part of the Fezouata Shale Formation, Morocco. Based on cephalic displacement and comparison to other trilobite moults, one specimen appears to represent a moulted exoskeleton. Four specimens are typical inter-moult carcasses. Four others are wrinkled and flattened, with thin exoskeletons compared to inter-moult specimens, and are considered post-moult individuals. These S. ebbestadi specimens illuminate the preservation and morphology of the post-moulting stage, characterised by strong anterior-posterior exoskeleton wrinkling, as well as overall body flattening and reduced visibility of thoracic articulations. Being found in the same lens, these specimens likely represent the first preserved in-the-act mass moulting event. The displayed sequence of moulting suggests the moulting process in trilobites was comparable to modern arthropods, and conserved within euarthropod evolutionary history.

Key words: Trilobita, mass moult, soft-shelled, post-moult, moulting, exoskeleton, Ordovician, Morocco.

Harriet B. Drage [harriet.drage@zoo.ox.ac.uk], Department of Zoology, University of Oxford, South Parks Road, OX1 3PS, UK; and Oxford University Museum of Natural History, Parks Road, OX1 3PW, UK. Thijs R.A. Vandenbroucke [thijs.vandenbroucke@ugent.be] and Peter Van Roy [Peter.VanRoy@ugent.be], Department of Geology, Ghent University, Campus 
Sterre, 9000 Ghent, Belgium. Allison C. Daley [allison.daley@unil.ch], Institute of Earth Sciences, University of Lausanne, Géopolis, CH-1015 Lausanne, Switzerland.

This is an open-access article distributed under the terms of the Creative Commons

Attribution License (for details please see creativecommons.org), which permits unrestricted use, distribution, and reproduction in any medium, provided the original author and source are credited.

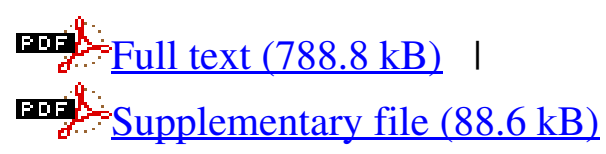

